\title{
Retrospective Evaluation of Risk Factors and Immunohistochemical Findings for Pre-Neoplastic and Neoplastic lesions of Upper Urinary Tract in Patients with Chronic Nephrolithiasis
}

\author{
Fanny Sharadkumar Desai ${ }^{1}$, Jitendra Nongthombam², Lisam Shanjukumar \\ Singh $^{1 *}$
}

\begin{abstract}
Background: Urinary stones are known predisposing factors for upper urinary tract carcinoma (UUTC) which are commonly detected at advanced stage with poor outcome because of rarity and lack of specific criteria for early detection. Aims and objectives: The main aim was to evaluate the impact of age, gender andstone characteristics on risk of developing UUTC in patients with chronic nephrolithiasis. We also discuss the role of aberrant angiogenesis (AA) and immunohistochemical expression of p53, p16INK4a, CK20 and Ki-67 in diagnosis of pelvicalyceal neoplastic (NL) and pre-neoplastic lesions (PNL) in these patients. Materials and Methods: Retrospective analysis of pelvicalyceal urothelial lesions from 88 nephrectomy specimens were carried out in a tertiary care centre from June 2012 to December 2014. Immunohistochemistry (IHC) was performed on 37 selected cases. Computed image analysis was performed to analyse aberrant angiogenesis. Results: All UUTC $(5.7 \%)$ and metaplastic lesions were found to be associated with stones. Some $60 \%$ were pure squamous cell carcinoma and $\mathbf{4 0 \%}$ were transitional cell carcinoma. Odd ratios for developing NL and PNL lesions in presence of renal stone, impacted stones, multiple and large stag horn stones were 9.39 (95\% CI 1.15-76.39, p value 0.05$), 6.28(95 \%$ CI 1.59-24.85, p value 0.000$)$ and $7.4(95 \% \mathrm{CI}, 2.29-23.94$, p value 0.001$)$ respectively. When patient age was $\geq 55$, the odds ratio for developing NL was 3.43 (95\% CI 1.19-9.88, p value 0.019). IHC analysis showed that mean Ki-67 indices were 3.15 $\pm 3.63 \%$ for non-neoplastic lesions, 10.0 $\pm 9.45 \%$ for PNL and 28.0 $\pm 18.4 \%$ for NL. Sensitivity and specificity of CK20, p53, p16INK4a, AA were 76\% and 95.9\%; 100\% and $27.5 \% ; \mathbf{1 0 0} \%$ and $26.5 \% ; 92.3 \%$ and $78.8 \%$ respectively. Conclusions: Age $\geq 55$ years, large stag horn stones, multiple stones and impacted stones are found to be associated with increased risk of NL and PNL in UUT. For flat lesions, a panel of markers, Ki 67 index $>10$ and presence of aberrant angiogenesis were more useful than individual markers.
\end{abstract}

Keywords: Upper urinary tract carcinoma - neoplastic lesions - pre-neoplastic lesions - renal stones

Asian Pac J Cancer Prev, 16 (18), 8293-8298

\section{Introduction}

Neglected renal stones are known predisposing factors for development of upper urinary tract carcinoma (UUTC). Several case reports and few studies have demonstrated association of UUTC with renal stones (Raghavendran et al.,2003; Yeh et al., 2007; Kim et al., 2009; Sun et al., 2013; Sahoo et al., 2015; Zengin et al., 2015). In India, higher incidences of squamous cell carcinoma (SCC) have been reported in stone associated malignancies (Raghavendran et al., 2003; Jain A et al., 2011). In Taiwan, transitional cell carcinoma (TCC) was found to be commonly associated with renal stones (Yeh et al., 2007; Sun et al., 2013). Adenocarcinoma also has been reported rarely (Raphael et al., 2011). The most likely reasons were stone induced irritation, infection, changes in local environment and subsequently leading to urothelial proliferation, metaplasia and malignant neoplasm. Pre-neoplastic lesions associated with stones have been described by very few studies (Beyer-Boon et al., 1978, Deniz et al., 2010; Hong et al., 2014; Zhou et al., 2014). These lesions are commonly missed because of nonspecific clinical presentation and conventional radiological findings due to presence of renal stones and therefore often detected at advanced stage with poor outcome (Raghavendran et al., 2003; Hong et al., 2014; Rheaume-Lanoie et al., 2014). Recently, European urological association (Roupret et al., 2013 ) has recommended Computed tomography urography (CTU)

${ }^{1}$ Department of Biotechnology, Manipur University, ${ }^{2}$ Department of Urology, Jawaharlal Nehru Institute of Medical Sciences, Imphal, India*For correspondence: shanjulisam@gmail.com 
and cytology followed by endoscopy to detect UUTC in patients with hematuria. Hematuria is present in majority of the patients with urinary stones (Cowan et al., 2012). CTU shows false positivity in presence of inflammation and renal stones (Sadow et al.,2010; Golan et al., 2015 ) and cytology examination is less sensitive ( Tai et al., 2015; Messer et al., 2011) due to associated reactive atypia. Though advancement in endoscopic instrument has lead to detection of UUTC at earlier stage, some lesions are still missing (Vashistha et al., 2013; Yamany et al., 2015 ). To date Specific criteria to guide early detection in patients with chronic nephrolithiasis in routine clinical practice are lacking and very few studies have described role of immunohistochemistry in UUTC, majority of them are prognostic makers (Chen s et al.,2013; Sasaki Y et al., 2014; Feng Ch et al., 2014). In Manipur, north eastern state of India, urinary stone is highly prevalent and neglected giant stag horn stones are commonly found (Singh et al., 1978; Marak et al., 2013) resulting in functional impairment of kidney and also predisposing factor for UUTC causing significant morbidity and mortality in the region. Present study was therefore conducted to evaluate impact of age, gender, stone characteristics on risk of developing UUTC in patients with chronic nephrolithiasis. We also discuss the role of aberrant angiogenesis and immunohistochemical expression of p53, p16INK4a, CK 20 and Ki-67 markers in diagnosis of pelvicalyceal neoplastic and pre neoplastic lesions in these patients.

\section{Materials and Methods}

A retrospective case controlled analysis of pelvicalyceal urothelial lesions from 85 nephrectomy and 3 nephroureterectomy specimens was conducted which underwent surgery for different reasons. Data were retrieved from histopathology and clinical records of a tertiary care centre from June 2012 to December 2014. Age, sex, stone characteristics, histopathology of urothelial lesions, angiogenesis and IHC expression were included in this study. All lesions were classified according to the World Health Organization /International Society of Urological Pathology (2004) (Ebel JN et al., 2004). In heterogeneous conditions, lesions that involved two grades or stages were classified according to the higher ones identified in the specimen. Tumour stage was determined according to the Union for International Cancer Control (UICC) 2009 TNM classification for urothelial cell carcinoma of the upper urinary tract (Sobin L. et al., 2009). History of past or present synchronous urinary bladder tumour was not found in any case. Nodal metastases of tumours were not included in this study due to incomplete clinical history.

\section{Immunohistochemistry}

IHC was performed on 37 selected cases representing all histopathologically classified lesions. Recommendations of best practices in immunohistochemistry in uro-pathology were followed (Amin et al., 2014 ). IHC performed by the avidin-biotin-complex method using Novolink polymer detection system (Novocastra, Newcastle, UK) on formalin-fixed, paraffin-embedded tissue cut into 4 micron sections. Primary ready to use antibodies were used against CK20 (Clone Ks20.8, Novocastra, Newcastle, UK), Ki-67 (Clone MM1, Novocastra, Newcastle, UK), p53 (Clone Do 7, Novocastra, Newcastle, UK) and p16INK4a (Biogenix, Forement, USA). For positive controls, we used cervical cancer and urinary bladder carcinoma and for negative controls, we omitted all primary antibodies. IHC expressions of multiple lesions from the same section were studied separately.

The abnormal expression of CK20 was concluded when it was expressed from the surface to middle layer or the whole layer and reported as negative and positive. The percentage of Ki67 positive cells was counted using image $\mathrm{J}$ cell counter on calibrated images and value was scored by labelling index. p53 and p16INK4a expressions were scored semi quantitatively as: negative (expressed in $<10 \%$ cells or weak patchy), weakly positive (full thickness) and strong positive (full thickness). All the lesions were reclassified into four categories: non-neoplastic, preneoplastic, and neoplastic non invasive and neoplastic invasive. Lesions were diagnosed on histopathology along with interpretation of IHC expression of panel of markers and were considered as gold standard (Amin et al, 2014). Strong and weak expression (full thickness) of p53 and/ or p16 INK4a with Ki 67 index $<10$ was considered as preneoplastic. Strong expressions of p53 and /or p16INK4a antibodies with $\mathrm{Ki} 67$ index $\geq 10$ were considered as neoplastic. Positive CK20 expression was considered as neoplastic and further classified according to morphology in low grade and high grade. Negative expressions of p16INK4a, p53 and CK 20 with or without high Ki 67 labelling index were considered as non neoplastic.

\section{Image analysis:}

Computed image analysis was performed to analyse aberrant angiogenesis (AA) in all urothelial lesions undergone IHC. AA was considered when number of vessels increased more than 10/hpf and/or increased moderate to large sized irregular, branching vessels immediately at the base of epithelium. Deeper lamina propria vessels were excluded from analysis.

\section{Statistical analysis:}

Statistical analysis was performed using IBM SPSS (version 21) software. We used Chi square, fisher exact tests and risk estimates for categorical variables while mean and Independent student ' $t$ ' test for continuous variables. Test was considered significant when $\mathrm{p}$ value was $<0.05$.

\section{Results}

H\&E stained paraffin embedded sections from 88 nephrectomy specimens were evaluated among which males were 22, females were 66. Median age for nephrectomy was 50 years with minimum 8 and maximum 75 years. Nephrectomy for stone disease was performed in 76 cases. Other cases were renal cell carcinoma (3), tuberculous pyelonephritis, (3), xanthogranulomatous pyelonephritis (4), and chronic end stage renal disease due to unknown reason (1). Non-functioning or poorly 
functioning kidneys on radiology was found in 72 cases. Ultrasound, intravenous pyelography were performed in all cases and computed tomography scan was performed in 20 cases. We found $30(34.1 \%)$ reactive urothelial atypia, one $(2.3 \%)$ inflammatory polyp, $6(6.8 \%)$ atypia of unknown significance, $11(12.5 \%)$ flat hyperplasia, $13(14.8 \%)$ papillary hyperplasia, $4(4.5 \%)$ squamous metaplasia, $1(1.1 \%)$ mucinous metaplasia, $8(9.1 \%)$ dysplasia, 4 (4.5\%) carcinoma in situ, 4 (4.5\%), low grade papillary lesions, $2(2.3 \%)$ high grade papillary urothelial carcinoma, and $3(3.4 \%)$ cases of squamous cell carcinoma on histopathology.

After IHC we found non neoplastic lesions in 52 $(59.01 \%)$ cases, preneoplastic (PNL) in 17 (19.32\%) cases, neoplastic lesions (NL) in $19(21.59 \%)$ cases. Among NL, $14(73.68 \%$ ) cases were non invasive (low grade 8 and high grade 6$)$ and $5(26.32 \%)$ cases were invasive. Among invasive lesions, $3(60 \%)$ were pure moderately differentiated SCC [Tumour stage T3 (1 case) and Stage T4 (2 cases)] and 2(40\%) were high grade TCC [stage T1 (1 case), stage T3 (1 case)]. One of the TCC showed squamous differentiation. One of the carcinomas was detected before operative procedure, 2 were diagnosed during endoscopic stone removal and 2 were diagnosed incidentally after histopathological examination. Frequency distribution of age, gender, size and number of stones, presence of stone impaction within urothelium, are presented in table 1 .

Renal stones were present in all urothelial carcinomas. Significant association was found between presences of stones, multiple stones, large stag horn stones, impacted stones and neoplastic, preneoplastic lesions. Odds ratio, Relative Risk and chi square $\mathrm{p}$ value are presented in table 2. We also found that the risk of NL was increased when age of patient was $\geq 55$ years. (chi square $\mathrm{p}$ value 0.019 . odd ratio 3.429 with $95 \%$ CI 1.190-9.877). Though PNL (mean age: 48vs 49) and NL (Mean age: 62 vs 65) presented at early age when stone was impacted, no definite statistical significance was found. We could not find statistical significance between gender and NL.

IHC was performed in 37 cases and total 78 lesions were studied immunohistochemically. Summary of the IHC expressions are presented in table 3. 26 (33.33\%) of cases were reclassified. flat hyperplasia, papillary hyperplasia, low grade papillary lesions and dysplasia were reclassified after IHC, while carcinoma in situ, high grade urothelial carcinoma, squamous dysplasia, squamous metaplasia and mucinous metaplasia were not reclassified (Fisher exact test $\mathrm{p}$ value, 0.000). Mean Ki 67 labelling indices were $28.02 \%$ and $3.03 \%$ in neoplastic and non neoplastic lesions respectively. (Independent' $t$ ' test, $\mathrm{p}$ value, $<0.001)$. Mean Ki 67 labelling index for low

Table 1. Frequency Distribution of Age, Gender, Size and Number of Stones, Stone Impaction

\begin{tabular}{|c|c|c|c|c|}
\hline & Non-Neoplastic & Pre-Neoplastic & Neoplastic non-invasive & Neoplastic Invasive \\
\hline No of cases=n $(\%)$ & $52(59.1)$ & $17(19.3)$ & $14(15.9)$ & $5(5.7)$ \\
\hline Mean (age) & 46 & 48 & 49 & 62 \\
\hline Standard Deviation & 14 & 15 & 12 & 6 \\
\hline \multicolumn{5}{|c|}{ Independent ' $t$ ' test $p$ value when compared with neoplastic invasive } \\
\hline & 0.014 & 0.057 & 0.034 & - \\
\hline \multicolumn{5}{|l|}{ Age with cut off value } \\
\hline$<55(\%)$ & $37(42.05)$ & $9(10.23)$ & $7(7.95)$ & $0(0)$ \\
\hline$\geq 55(\%)$ & $15(17.05)$ & $8(9.09)$ & $7(7.95)$ & $5(5.68)$ \\
\hline \multicolumn{5}{|l|}{ Gender } \\
\hline Male (\%) & $15(17.05)$ & $1(1.14)$ & $5(5.68)$ & $1(1.14)$ \\
\hline Female $(\%)$ & $37(42.05)$ & $16(18.18)$ & $9(10.23)$ & $4(4.55)$ \\
\hline \multicolumn{5}{|l|}{ Presence of stones } \\
\hline Multiple and large stones (\%) & $27(30.68)$ & $13(14.77)$ & $14(15.91)$ & $5(5.68)$ \\
\hline Small stones $(<15 \mathrm{~mm})(\%)$ & $14(15.91)$ & $3(3.41)$ & $0(0)$ & $0(0)$ \\
\hline No stones $(\%)$ & $11(12.5)$ & $1(1.14)$ & $0(0)$ & $0(0)$ \\
\hline \multicolumn{5}{|c|}{ Stone impaction within urothelium } \\
\hline Present $(\%)$ & $3(3.40)$ & $2(2.27)$ & $4(4.55)$ & $4(4.55)$ \\
\hline Absent $(\%)$ & $49(55.68)$ & $15(17.05)$ & $10(11.36)$ & $1(1.14)$ \\
\hline
\end{tabular}

Table 2. Risk Estimates of Neoplastic and Pre Neoplastic Lesions

\begin{tabular}{lccc}
\hline Risk factor & $\begin{array}{c}\text { Odds Ratio } \\
(95 \% \mathrm{CI})\end{array}$ & $\begin{array}{c}\text { Relative Risk } \\
(95 \% \mathrm{CI})\end{array}$ & $\begin{array}{c}\text { Chi-square } \\
\mathrm{p} \text { value }\end{array}$ \\
\hline Presence of stones (NL)** & $-*$ & $1.21(1.09-1.35)$ & 0.05 \\
Presence of stone Impaction (NL) & $9.31(2.57-33.73)$ & $5.81(2.15-15.72)$ & 0 \\
Presence of multiple and large stag horn stones (NL) & $-*$ & $1.73(1.41-2.11)$ & 0.001 \\
$\geq 55$ age (NL) & $3.43(1.19-9.88)$ & $1.9(1.17-3.06)$ & 0.019 \\
Presence of stones (PN + NL)*** & $9.39(1.15-76.39)$ & $1.23(1.06-1.43)$ & 0.014 \\
Presence of stone Impaction (PN+ NL) & $6.28(1.59-24.85)$ & $4.82(1.42-16.28)$ & 0.004 \\
Presence of multiple and large stag horn stones (PN+NL) & $7.4(2.29-23.94)$ & $1.71(1.29-2.28)$ & 0 \\
$\geq 55$ age (PN+ NL) & $3.08(1.25-7.51)$ & $1.93(1.15-3.23)$ & 0.012 \\
\hline
\end{tabular}

*not counted due to presence of zero value in one cell, **Risk estimates for NL: Neoplastic, *** Risk estimates for NL+PNL: neoplastic and preneoplastic 
Table 4. Role of Individual Marker in Detecting Neoplastic Lesions

\begin{tabular}{lcccc}
\hline Marker & $\begin{array}{c}\text { Sensitivity } \\
(\%)\end{array}$ & $\begin{array}{c}\text { Specificity } \\
(\%)\end{array}$ & $\begin{array}{c}(+) \\
\text { predictive } \\
\text { value (\%) }\end{array}$ & $\begin{array}{c}(-) \\
\text { predictive } \\
\text { value (\%) }\end{array}$ \\
\hline CK20 & 76 & 95.9 & 90.5 & 88.7 \\
p53(wild type) & 100 & 27.5 & 41.3 & 100 \\
p16INK4a & 100 & 26.5 & 41 & 100 \\
Ki 67 labelling Index $\geq 10$ & 84.6 & 67.3 & 56.4 & 89.7 \\
Ki 67 labelling Index $\geq 25$ & 53.8 & 94.2 & 82.4 & 80.3 \\
Ki 67 labelling Index $\geq 30$ & 46.2 & 98.1 & 92.3 & 78.5 \\
Aberrant angiogenesis & 92.3 & 78.8 & 68.6 & 95.3 \\
\hline
\end{tabular}

grade and high grade neoplastic lesions were $13 \%$ and $38 \%$ (Independent ' $t$ ' test $\mathrm{p}$ value 0.002 ).

Aberrant angiogenesis was found in 35 lesions out of 78 lesions studied immunohistochemically and more commonly found in NL (24/35) and PNL (11/35) (Fisher exact test $\mathrm{p}$ value ,0.000). Sensitivity, specificity and predictive values of different markers in diagnosis of neoplastic lesions are presented in table 4 .

\section{Discussion}

Association between long standing urinary stones and upper urinary tract carcinoma is well known. Very few studies have described spectrum of upper urinary tract lesions associated with chronic stone disease (Beyer et al., 1978; Hong et al, 2014). However to date, statistical evaluation of risk factors associated with increased risk of neoplastic and pre-neoplastic UUT lesions in patients with chronic nephrolithiasis and role of immunohistochemical expressions of p53, CK20, Ki67 and p16INK4a in diagnosis of these lesions have not been evaluated before.

Our study shows significant association between presence of kidney stone, size of the stones and stone impaction within urothelium and increased risk of UUTC. All urothelial carcinoma were associated with stones. SCCs $(60 \%)$ were more common than TCCs with M:F ratio 1:3. Our findings are similar to other series from India (Raghavendran et al., 2003; Jain et al., 2011) and Turkey (Zengin et al, 2015) except, these series found male predominance. However in Taiwan, Yeh et al. (2007) and Sun et al.(2013) found that TCC were more common in patients with stone disease with female predominance.

Cheungpasitporn et al. (2015) found pooled relative risk of TCC in patients with kidney stones was 2.14. (95\% CI 1.35-3.40) and Sun et al. (2013) found Hazard Ratio 4.66 (95\% CI 2.97-7.30) for UUTC in patients with urinary stones. In our study, relative risk for developing neoplastic lesions in patients with renal stone was 1.211 (95\% CI 1.086-1.349). When stones were large (stag horn) and multiple, this risk increased 1.7 fold and with impacted stones this risk increased 5.8 fold.

We found statistically significant differences in mean age for non neoplastic, preneoplastic lesions, neoplastic non invasive and invasive neoplastic lesions which were 46, 48, 49 and 62 years respectively. Mean age for superficially invasive and non invasive NL was 50 years while deeply invasive (stage 3 and 4) mean age was 64 years (Independent ' $t$ ' test, $p$ value 0.025 ). These findings 
suggest that it takes $13-14$ years to develop deeply invasive neoplasm from non-invasive neoplastic and pre neoplastic lesions. We found that when age of patient is $\geq 55$ years, the risk of finding NL increases 3 fold (Chi square $\mathrm{p}$ value 0.019 .). Mean age for squamous metaplasia was 50.43 , for squamous dysplasia 58 and for squamous cell carcinoma 62.5. Our findings supports that once the dysplasia is developed in squamous lesions, progression to squamous carcinoma is rapid.

Due to presence of inflammation and associated reactive changes in chronic stone disease and lack of interobserver agreement (Murata et al., 2010) for flat urothelial lesions, we did immunohistochemical analysis when it was difficult to diagnose just on the basis of morphology alone.( Amin et al, 2014). Each marker's diagnostic ability is presented in table 4, which shows that panel of markers were more useful than individual marker in diagnosis of NL.All NL showed strong p53 and p16INK4a expression and $\mathrm{Ki} 67$ labelling index $\geq 10$. High grade lesions showed mean Ki 67 index $38 \%$ and majority were classified correctly on histopathology alone (Fisher exact $\mathrm{p}$ value < 0.001). Like findings of Hammam O et al (2014) and Gaisa et al (2011) in urinary bladder, negative CK20 expression was found in all squamous cell carcinoma, squamous cell carcinoma in situ and squamous dysplasia and positive CK20 expression found in neoplastic urothelial lesions as well as non-neoplastic glandular metaplasia. We found expression of wild type p53 (weak as well as strong expression) and p16INK4a expressions even in the lesions which are not neoplastic morphologically, in flat hyperplasia, papillary hyperplasia, metaplasia and few cases of atypia of unknown significance. Low grade papillary urothelial lesions showed expression of all markers. Papillary hyperplasia, flat hyperplasia and metaplastic lesions are considered as PNL in urinary bladder ( Montironi et al, 2008; Readal and Epstein et al, 2010 ). We found week or strong p53 and or p16 INK4a expressions in these lesions in UUT without high ki 67 index. These markers associated genes were found to be altered in early pathways of carcinogenesis in genetically engineered mice (Wu X et al, 2009; Zhou et al 2012) Thus, In UUT also these lesions should be considered as preneoplastic and follow up is warranted. Low grade papillary lesions should be considered as neoplastic lesions.

Our study also demonstrates that AA was more commonly found in NL and PNL. (Fisher exact $\mathrm{p}$ value, $0.000)$. This is due to tumour neo-vasculature differs significantly from normal vascular development, a process that is not well understood but may be due to different VEGF-A isoforms expressions ( Nagy et al., 2009). Detection of neovascularisation during endoscopic stone removal helped us to search for urothelial carcinoma and two urothelial carcinoma and one carcinoma in situ were diagnosed on endoscopic biopsy. Role of narrowband imaging in identifying NL in upper urinary tract can be a cheaper and confirmative approach in patient with chronic nephrolithiasis and need to be evaluated further. Aberrant angiogenesis on histopathology can identify NL and separates these entities from non-neoplastic lesions and can be evaluated and incorporated in criteria to classify flat lesions of urothelium.

There are some limitations of our study. Not all patients with stones develop urothelial carcinomas and in many patients PNL were resolved after stone removal (BeyerBoon et al., 1978; Katz et al., 2005). Evaluation of utility of risk factors and different markers in clinical practice needs prospective studies. We did not evaluate hematuria because of incomplete history and its doubtful value in patients with chronic stone disease as majority of them present with hematuria. CTU though recommended, we could not perform due to cost factors and lack of expertise. Another limitation was we evaluated aberrant angiogenesis morphologically without endothelial markers due to limited study material. We omitted recommended CD 44s marker in differential diagnosis panel as its expression in squamous lesions are not well described.

In short, we described statically significant association between age $\geq 55$ years, multiple stones, large stones and impacted stones and increased risk of neoplastic and pre-neoplastic lesions in UUT. Presence of Aberrant angiogenesis, Ki 67 index $>10$ and IHC panel of p53/ p16INK4a and CK20 markers are more useful than individual marker in diagnosis of neoplastic lesions of UUT. High grade lesions were identified correctly on histopathology alone. Our findings can be helpful in monitoring and early detection of UUTC in patients with chronic nephrolithiasis.

\section{References}

Amin M, Epstein J, Ulbright $T$ et al (2014). Best Practices Recommendations in the Application of Immunohistochemistry in Urologic Pathology Report From the International Society of Urological Pathology Consensus Conference. Am J Surg Pathol, 38, 1017-22

Beyer-Boon ME, Cuypers LH, de Voogt HJ, Brussee JA (1978). Cytological changes due to urinary calculi: a consideration of the relationship between calculi and the development of urothelial carcinoma. Br J Urol, 50, 81-9

Chen S, Wu W, Tu H et al (2013). Glutathione S-transferase expression in upper urinary tract urothelial carcinomas: $a$ Taiwan Study Asian Pac J Cancer Prev, 14, 6475-9

Cheungpasitporn W, Thongprayoon C, O'corragain OA et al (2015). The risk of kidney cancer in patients with kidney stones: a systematic review and meta-analysis. QJM: An International Journal of Medicine, 108, 205-12

Cowan NC (2012). CT urography for hematuria. Nat Rev Urol, 13, 218-26.

Deniz K, Kala M (2010). Intestinal Metaplasia of the Renal Pelvis. Urol J, 7, 287-9.

Eble JN, Sauter G, Epstein JI et al (2004). WHO classification of tumours: pathology and genetics of tumours of the urinary system and male genital organs. IARC Press, Lyon, France, 111-2

Epstein J (2010).Diagnosis and Classification of Flat, Papillary, and Invasive Urothelial Carcinoma: The WHO/ ISUPConsensus. International Journal of Surgical Pathology, 18, 106-11

Feng Ch, Wang L, Ding G et al (2014). Predictive value of clinicopathological markers for the metachronous bladder cancer and prognosis of upper tract urothelial carcinoma. SCIENTIFIC REPORTS, 4.

Gaisa NT, Braunschweig T, Reimer N et al (2011). Different 
immunohistochemical and ultrastructural phenotypes of squamous differentiation in bladder cancer. Virchows Arch, 458, 301-12.

Golan S, Nadu A, Lifshitz D (2015). The role of diagnostic ureteroscopy in the era of computed tomography urography. BMC Urology, 15.

Hartmann A, Moser K, Kriegmair M (1999). Frequent Genetic Alterations in Simple Urothelial Hyperplasias of the Bladder in Patients with Papillary Urothelial Carcinoma. The American Journal of Pathology, 154, 721-7

Hong S, Kwon T, You D et al (2014). Incidence of benign results after laparoscopic radical nephroureterectomy. JSLS, 18, 335.

Jain A, Mittal D, Jindal A et al (2011). Incidentally detected squamous cell carcinoma of renal pelvis in patients with staghorn calculi: case series with review of the literature. ISRN Oncol.

Katz R, Gofrit ON, Golijanin D et al (2005). Urothelial cancer of the renal pelvis in percutaneous nephrolithotomy patients. Urol Int, 75 , 17-20

Kim M, Chung J, Hwang I et al ( 2009). Stag horn Stones Combined with Transitional Cell Carcinoma of the Renal Pelvis. Korean Journal of Urology, 50, 1027-31

Marak A, Shantibala K, Singh A, Singh N, Singh S (2013). Urolithiasis prevalence and related factors in a rural area of Manipur. Int J Med Sci Public Health, 2, 956-9

Messer J, Shariat SF, Brien JC, et al (2011). Urinary cytology has a poor performance for predicting invasive or high grade upper-tract urothelial carcinoma. BJU Int, 108, 701-5

Montironi R, Lopez-Beltran A, Scarpelli M, Mazzucchelli R, Cheng L (2008). Morphological classification and definition of benign, preneoplastic and non-invasive neoplastic lesions of the urinary bladder. Histopathology, 53, 621-33

Murata Sh, Iseki M, Mitsuru Kinjo M et al (2010). Molecular and Immunohistologic Analyses Cannot Reliably Solve Diagnostic Variation of Flat Intraepithelial Lesions of the Urinary Bladder. Am J Clin Pathol, 134, 862-72

Nagy JA, Chang S-H, Dvorak AM, Dvorak HF (2009). Why are tumour blood vessels abnormal and why is it important to know?. British Journal of Cancer, 100, 865 -9

Raghavendran M, Rastogi A, Dubey D, et al (2003). Stones associated renal pelvic malignancies. Indian J Cancer, $\mathbf{4 0}$, 108-12

Raphael V, Sailo S, Bhuyan A, Phukan M (2011). Mucinous adenocarcinoma of the renal pelvis with adenocarcinoma in situ of the ureter. Urol Ann, 3, 164-6

Readal N, Epstein JI (2010). Papillary urothelial hyperplasia: relationship to urothelial neoplasms. Pathology, 42, 360-3

Rhéaume-Lanoie J, Lepanto L, Fradet V, Billiard JS, Tang A (2014). Diagnostic performance of ultrasound for macroscopic hematuria in the era of multidetector computed tomography urography. Can Assoc Radiol J, 65, 253-9

Rouprêt M, Babjuk M, Compérat E et al (2013). European guidelines on upper tract urothelial carcinomas: 2013 update. Eur Urol, 63, 1059-71

Sadow CA, Wheeler SC, Kim J, Ohno-Machado L, Silverman SG (2010). Positive predictive value of CT urography in the evaluation of upper tract urothelial cancer. Am J Roentgenol, 195, 337-43

Sahoo T, Das S, Mishra C, et al (2015). Squamous Cell Carcinoma of Kidney and Its Prognosis: A Case Report and Review of the Literature. Case Rep Urol.

Sasaki Y, Sasaki T, Kawai T et al (2014) HER2 protein over expression and gene amplification in upper urinary tract urothelial carcinoma-an analysis of 171 patients. Int J Clin Exp Pathol, 7, 699-708

Singh PP, Singh LBK, Prasad S, Singh M (1978). Urolithiasis in Manipur (north eastern region of India). Incidence and chemical composition of stones. The American Journal of Clinical Nutrition, 31, 1519-25

Sun LM, Lin CL, Chang YJ et al (2013). Urinary tract stone raises subsequent risk for urinary tract cancer: a population-based cohort study. BJU Int, 112, 1150-5

Sobin LH, Gospodarowicz MK, Wittekind Ch (2009). [Renal Pelvis and ureter] TNM classification of malignant tumours. Urological tumours. International Union Against Cancer. Wiley-Blackwell, New York

Tai Y, Chiang I, Huang Ch, Tai H, Pu Y (2015). Effectiveness of different diagnostic tools for upper urinary tract urothelial carcinoma. Urological Science, 26, 57-60

Vashistha V, Shabsigh A, Zynger D (2013). Utility and diagnostic accuracy of ureteroscopic biopsy in upper tract urothelial carcinoma. Archives of Pathology \& Laboratory Medicine, 137, 400-7

Wu X (2009). Biology of urothelial tumorigenesis: insights from genetically engineered mice. Cancer Metastasis Rev, 28, 281-90

Yamany T, van Batavia J, Ahn J, Shapiro E, Gupta M (2015). Ureterorenoscopy for upper tract urothelial carcinoma: how often are we missing lesions?. Urology, 85, 311-5.

Yeh CC, Lin TH, Wu HC et al (2007). A high association of upper urinary tract transitional cell carcinoma with nonfunctioning kidney caused by stone disease in Taiwan. Urol Int, 79, 19-23.

Zengin K, Tanik S, Sener NC et al (2015). Incidence of renal carcinoma in non-functioning kidney due to renal pelvic stone disease. Molecular and Clinical Oncology, 3, 941-3

Zhou H, Huang H, Shapiro E et al (2012).Urothelial tumour initiation requires deregulation of multiple signalling pathways: implications in target-based therapies. Carcinogenesis, 33, 770-80

Zhou W, Zhong K, Wang J et al (2014). Intestinal metaplasia of the renal pelvis: A case report and literature review. ONCOLOGY LETTERS, 8, 2664-8 